\title{
Road Nail: Intelligent Road Marking System Testbed
}

\author{
Dragan Samardzija, Erne Kovac, Djordje Isailovic, Bojan Miladinovic, Nikola Teslic, Mihajlo Katona \\ University of Novi Sad \\ Novi Sad, Serbia \\ dragan.samardzija, nikola.teslic, mihajlo.katona@rt-rk.uns.ac.rs \\ erne.kovac, djordje.isailovic, bojan.miladinovic@rt-rk.com
}

\begin{abstract}
In this paper we present Road Nail, an intelligent road marking system with the wireless network of signaling devices that are mounted near the road edge. "Nails" or signaling devices are autonomous nodes with capability to accumulate electrical energy into battery which is obtained from solar panels. In addition, the nodes exchange wireless messages, detect approaching vehicles and emit signalization light. We have built an experimental testbed that consists of 20 nodes and a cellular gateway. In this paper, implementation details of the above system, including extensive measurements and performance evaluations in realistic field deployments are presented.
\end{abstract}

Index Terms - sensors, smart roads, V2I, wireless network

\section{INTRODUCTION}

Driving in low visibility conditions (e.g., fog or heavy precipitation), and during night, is particularly challenging leading to increased probability of traffic accidents and possible injuries. In this paper we present a system for intelligent marking of the road edge during low visibility and night-time. The system is known as Road Nail.

Recently, extensive research and a number of innovations in the field of improved road safety have been reported (e.g., [1, 2]). In addition, rapid improvements and wide application of semiconductor products have been the key enablers of effective implementation of sensor, wireless and battery technologies [3].

In this study we combine the most advanced solutions in sensor, wireless communication and battery-powered technologies to design and implement the novel road-safety system. It consists of an array of nodes that are installed on both sides of the road, being mutually separated by a few tens to a few hundreds of meters. Each node consists of (i) lighting subsystem for signalization, (ii) sensor subsystem for vehicle detection, (iii) wireless communication subsystem for message exchange between the neighboring nodes, and (iv) energy-supply subsystem with a battery and solar panels. During the day, nodes have their batteries recharged, while during low visibility and/or night they transition to active mode enabling detection of passing vehicles and timely activation of the signalization. Upon detection of a passing vehicle, the node generates a wireless message. After the detection or reception of a message indicating that a nearby node detected a vehicle, the signalization lights should be turned on (either red or white depending on the side of the road).

This work was partially supported by the Ministry of Science and Technological Development of Serbia under the project No. 11005, year 2008.
In order to save battery power the lights will be turned off after a predefined period of time. The nodes are installed on the side of the road, not requiring disruption of traffic during the installation and maintenance. Furthermore, this system may be connected to the global Internet via a specific gateway. This would allow local node information to be forwarded to a remote control center where road and node conditions may be monitored. This represents a very versatile implementation of a vehicle-to-infrastructure (V2I) system.

The system may eventually replace the existing signalization that is based on passive reflectors. We believe that application of this system would dramatically improve the traffic road safety. Considering the total length of the US motorways $(4,209,835$ kilometers [4]), there is a clear market potential behind this and similar solutions.

In Section II we describe the network organization, installation and functionality. In Section III the node architecture is described, with features and performance of each of the subsystems. In Section IV integration with the Internet is described, including a gateway coverage analysis. We conclude in Section V.

\section{OUTLINE OF NETWORK ORGANIZATION AND SYSTEM FUNCTIONALITY}

Figure 1 illustrates the basic installation and functionality of the system. The distance between the nodes depends on the conditions of the road. For example, sharper curves would require denser installation of the nodes. We have implemented a testbed that consists of 20 Road Nail nodes.

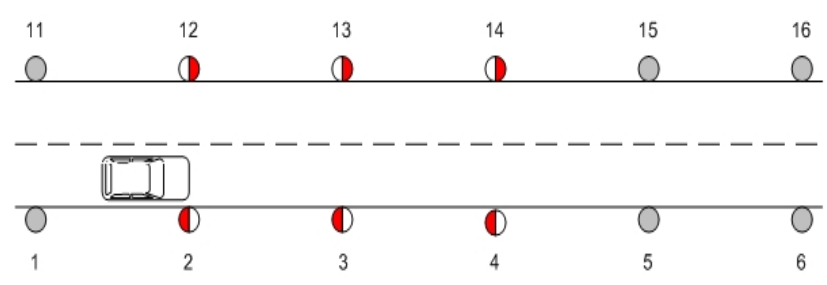

Figure 1. Road Nail system.

As given in Figure 1, a vehicle is detected for the first time by node 1 after which the system transitions from the low power consumption mode to the fully operational mode. After the vehicle has been detected by node 1 , it turns the red signalization light on, creates appropriate message and transmits it to node 2 in the direction of the vehicular movement. It also sends the message to its pair node 11 on the opposite side of the road to also switch on 
its signalization white light. Upon the reception of the message, node 2 turns the red signalization light on, sends wireless message to its pair node 12 and the node 3 . As soon as the vehicle passes by node 1 , in order to conserve energy, it turns off its signalization light. The process continues in the direction of the vehicular movement

The protocol is designed such that the communication distances are maximized, with timely transmission of the messages and with the lowest possible power consumption. In addition, the protocol is solving the problem of coupling between physical locations of the nodes and their network addresses. A node is turning its signalization on only if its immediate neighbors have generated message, while ignoring messages received from the nodes which may be at a greater distance. This is actually a topology-discovery problem which is beyond the scope of this paper.

\section{Signaling DeVICE ARChitecture}

Each signaling device in the Road Nail system consists of four subsystems, as shown in Figure 2.

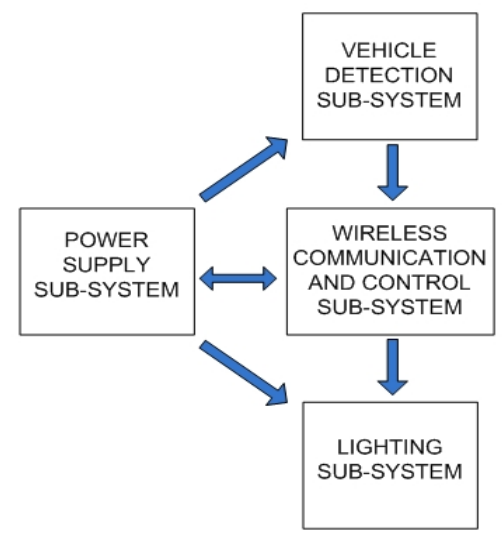

Figure 2. Block diagram of signaling device.

The Road Nail node implementation is shown in Figure 3. The upper part of the signaling device (4) is made of transparent, waterproof material (e.g., glass) allowing passing of the solar light to the solar cell (6) and protecting electronic components $(7,8,9$ and 10) from rain or snow. On the front and back side of the housing, there are LED light sources (2 and 12), with reflective backgrounds ( 3 and 11). As given in Figure 3, the nodes may be installed such that the lower part of the device (1) is in the ground (5). Alternatively, the nodes may also be installed above the ground (e.g., typically $80 \mathrm{~cm}$ above the ground), where additional mechanical support structure is needed. Inside the housing there is a printed circuit board with microcontroller and subsystem for wireless communication (7), a number of sensors (8) and battery (9) and its management (10).

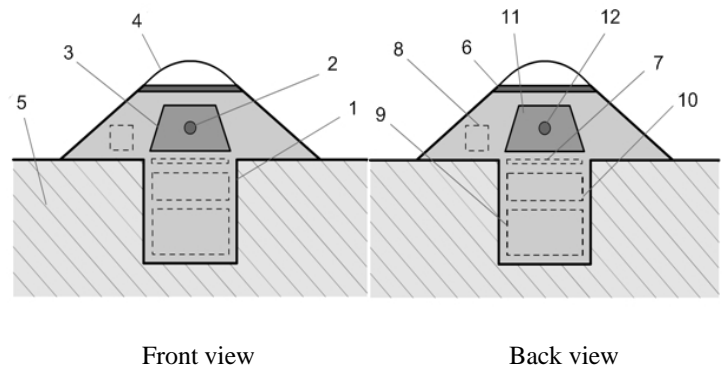

Figure 3. Road Nail node.

\section{A. Vehicle detection subsystem}

The sensor, i.e., vehicle detection subsystem consists of a set of sensors which provide information to the wireless communication control subsystem. In this particular implementation we applied a passive infrared (PIR) sensor Parallax 555-28027 for movement detection. This particular sensor has been proven very efficient with the detection range of approximately 15 meters, and low power consumption of less than $15 \mathrm{~mW}$. For example, in Figure 4 we present the output voltage as a function of time where vehicles passed at $t=3,9,14,26,36,40,49$ and 58 seconds. Extensive measurements indicate very high probability of correct detection. The measurements are performed in realistic conditions.

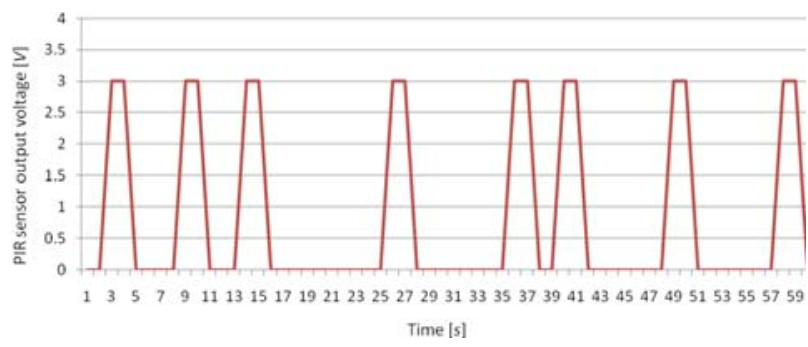

Figure 4. PIR detector output as a function of time with vehicles passing at $t=3,9,14,26,36,40,49$ and 58 seconds.

In addition, we applied a light sensor Intersil ISL29003 for detection of vehicle headlights. Its power consumption is approximately $1 \mathrm{~mW}$. Extensive measurements confirmed very high probability of successful vehicle detection. As an example, in Figure 5 we present the sensor output as a function of an approaching vehicle distance, with the headlights on.

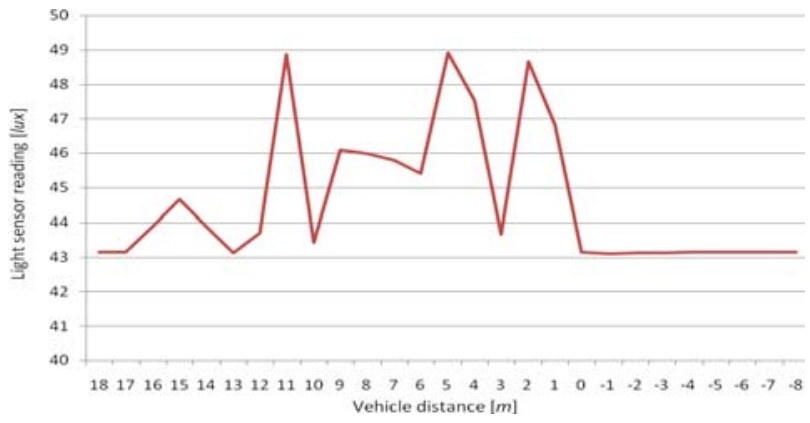

Figure 5. Light sensor output as a function of distance between the passing vehicle and the node. 


\section{B. Lighting subsystem}

The lighting subsystem consists of a set of LEDs and an LED driver. The LEDs are applied because of their low power consumption while having satisfying brightness providing a clear and visible marking of the road edge in low visibility. Based on the extensive analysis and measurements we conclude that the lighting system consumes a significant fraction of the available power, namely, it accounts for more that $60 \%$ of overall power consumption (Figure 6).

\section{Wireless communication and control subsystem}

Wireless communication and control subsystem is based on a wireless transceiver and 8051 micro-processor core, all integrated in Texas Instruments CC2530. It is in charge of exchanging messages over the wireless interface, as well as data sensor analysis and battery state control. The wireless network interface is based on the IEEE 802.15.4 standard [5] which defines the physical and medium access control layer for personal area network (PAN). The standard uses carrier sense multiple access with collision avoidance medium access (CSMA/CA) mechanism and supports star as well as peer-to-peer topologies. The implemented protocol ensures features like low complexity, cost, power consumption, including low wireless data rates. Low power consumption is the most important feature in this application due to limited amount of accumulated energy. It is achieved with a stand-by feature which provides possibility for every device to go into the sleep mode with ultra low power consumption during the day or when there are no vehicles. Under the typical conditions, this subsystem consumes approximately $25 \%$ of overall power.

\section{Power supply subsystem}

The power supply subsystem consists of the following three segments: solar cells, rechargeable battery and battery management. The solar cells provide enough energy for full functionality of the signaling device over some predefined period of time. The battery management is in charge of monitoring and control of all battery parameters including battery charging and discharging. We have conducted a number of laboratory and field measurements. The power consumption measurement results (per each subsystem and total) are presented in Figure 6. The measurements are performed during night time, at around $15^{\circ} \mathrm{C}$. For the given power consumption, we have analytically and experimentally confirmed that fully charged battery could support almost 20 hours of the continuous full operation (without being recharged during that period).

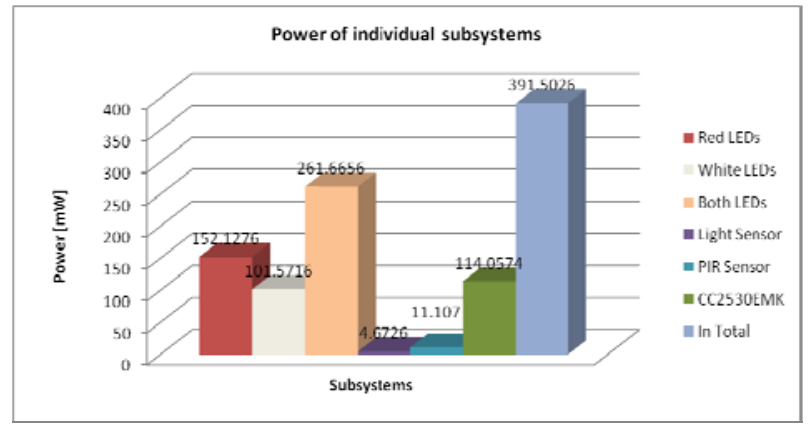

Figure 6. Power consumption measurement results.

In order to meet projected power consumption three solar modules SOL4 Vellerman are applied each $66 \times 95 \times 6$ millimeters. Extensive measurements have been performed during cloudy or sunny atmospheric conditions, with different sun incidence angles. Examples of the measurements are given in Figure 7 and 8, depicting generated current and power, as a function of voltage.

Our analysis indicates that in order to have continuously operational system, in a period lasting four days, it is sufficient to have aggregate of 10 sunny hours enabling proper recharging of the battery (on average, 2.5 sunny hours a day).

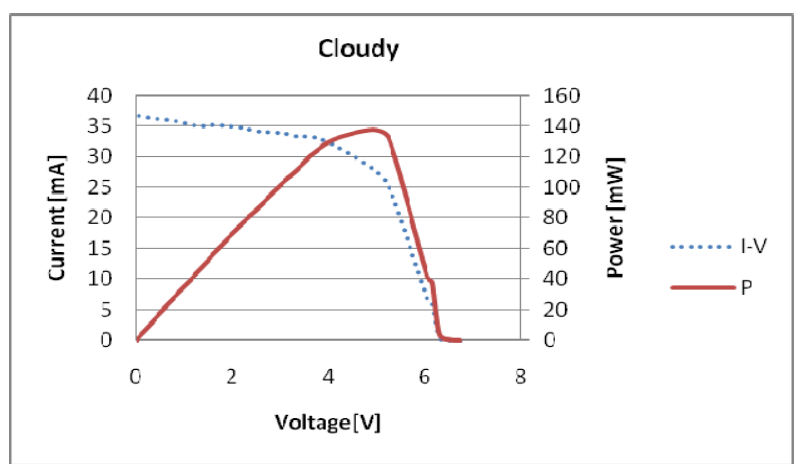

Figure 7. Solar cell power generation, cloudy day, $-4{ }^{\circ} \mathrm{C}$.

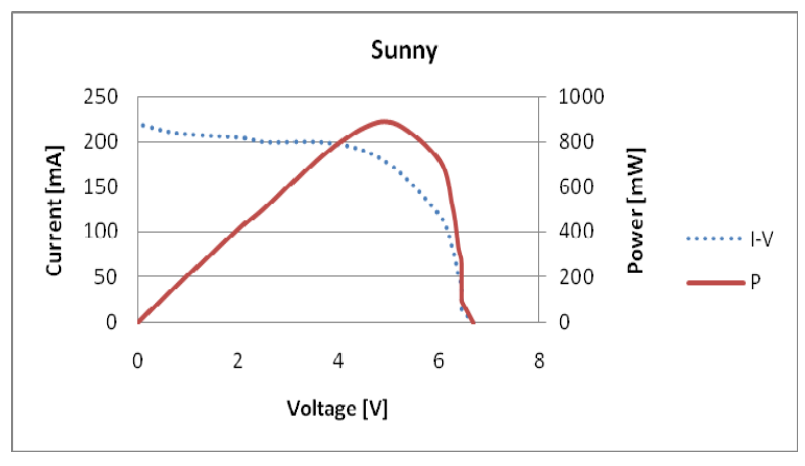

Figure 8. Solar cell power generation, sunny day, $1^{\circ} \mathrm{C}$, sun incidence angle $45^{\mathrm{O}}$. 


\section{INTEGRATION WITH CELLULAR NETWORK AND THE INTERNET}

Figure 9 depicts integration of the Road Nail system with the cellular network and the Internet. The integration has been realized as a part of the testbed. In addition to the IEEE 802.15.4 wireless network between the nodes, a wireless link between the system and the Internet is established via a gateway and the cellular network (e.g., GPRS/EDGE, UMTS or LTE). In this way information that is local to the nodes is available to a remote control center. The information may be used for

- traffic emergency monitoring (e.g., a car is stalled or crash is suspected),

- speed control,

- traffic monitoring (e.g., cars are slowing down, indicating congestion),

- road maintenance (e.g., cars are slowing down, indicating a pothole),

- road planning (e.g., detected increase in traffic),

- emergency push button,

- node maintenance required...

Furthermore, the control center can send different information to all or individual nodes in the area. The nodes could transmit the received information to passing vehicles. For example,

- traffic conditions,

- weather,

- work and special condition,

- advised minimal distance between vehicles...

The above system represents one versatile implementation of the V2I concept.

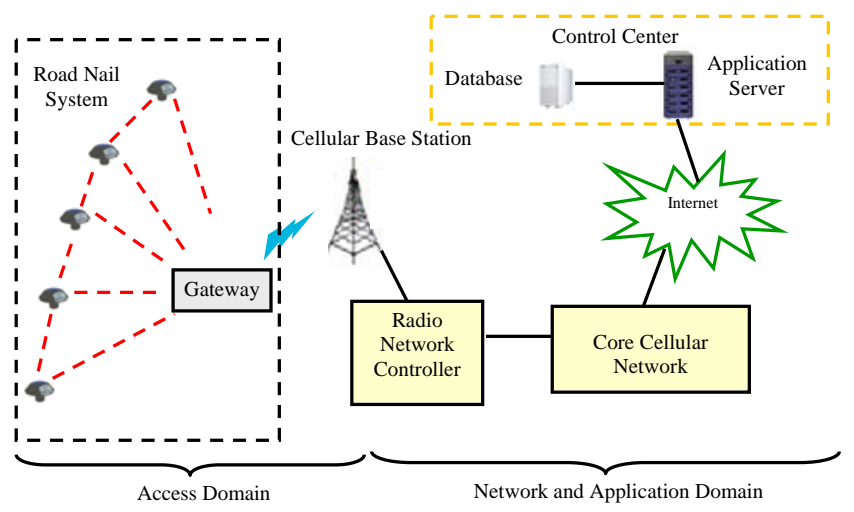

Figure 9.Road Nail system integrated with the Internet and the control center.

\section{A. Gateway and Coverage Problem}

The gateway integrates two wireless interfaces: (i) the 802.15.4 interface connecting to individual Road Nail nodes, and (ii) cellular interface connecting to the neighboring cellular base station. The gateway is servicing a particular geographic area whose size is primarily limited by the short-range gateway-to-node coverage.

In order to asses the coverage, we first consider a case where a direct link, i.e., a single hop, is established between a node and the gateway. We will assume the minimum receiver sensitivity required by the IEEE 802.15.4 standard, $P_{\text {rsen }}=-85 \mathrm{dBm}$. The maximum node transmit power is set to a typical value $P_{\text {tmax }}=0 \mathrm{dBm}$. The $3 \mathrm{GPP}$ path loss model is applied, Release 9, TR 36.814 V2.0.1 [6-8]. The node height is $h_{n}=1$ meter, and the gateway height $h_{g w}=10$ meters. Both line-of-sight (LOS) and non-line-of-sight (NLOS) cases are considered. The coverage, i.e., probability, that the received power exceeds the receiver sensitivity $P_{r s e n}$, as a function of distance between the node and the gateway, is presented in Figure 10. From the results, we note a very limited coverage with the distances presented in Table I.

In addition, a multi-hop scenario is also considered. In this case, a message from a node may be relayed by intermediate nodes on its way to the gateway. Number of hops and relays involved are selected out of all possible combination such that the coverage is maximized (i.e., probability that the received power exceeds the receiver sensitivity threshold is maximized). To model the channel between the nodes, i.e., relays, we apply the model in [9], with an additional loss to due to the $2.4 \mathrm{GHz}$ carrier frequency. The corresponding results are presented in Figure 11. Different node densities are considered, where nodes are uniformly distributed in the area of radius $R$. We note improvement in coverage over the no-relaying, i.e., single-hop case (Table II). The gain increases with the node density, i.e., number of nodes in the given area.

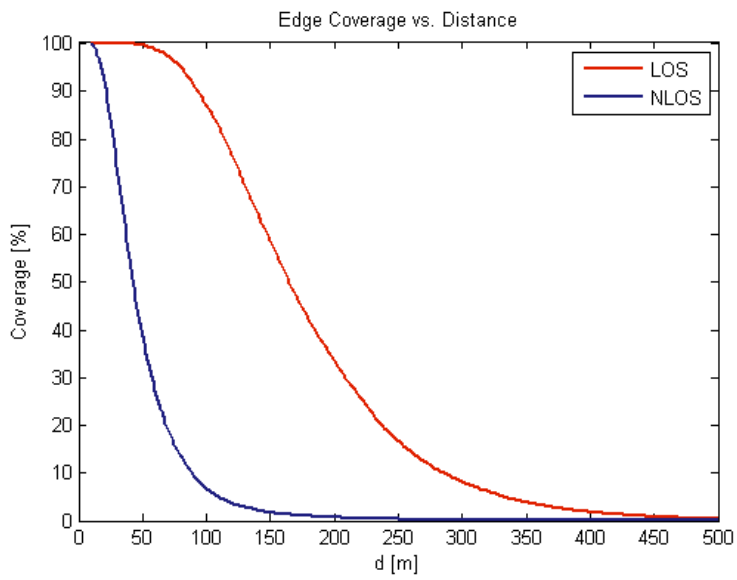

Figure 10. Single-hop coverage as a function of distance.

Table I. Single-hop coverage.

\begin{tabular}{|c|c|c|}
\hline & $90 \%$ coverage & $99 \%$ coverage \\
\hline LOS & $90 \mathrm{~m}$ & $50 \mathrm{~m}$ \\
\hline NLOS & $20 \mathrm{~m}$ & $10 \mathrm{~m}$ \\
\hline
\end{tabular}

According to the Shannon capacity formula, for the 802.15.4 data rates and bandwidth, the lower bound on the receiver sensitivity is at $-114.28 \mathrm{dBm}$. Consequently, the gap between the lower bound and the 802.15.4 receiver sensitivity is $29.28 \mathrm{~dB}$. While the lack of channel coding in 
802.15.4 may account for approximately $8 \mathrm{~dB}$, the rest may be attributed to lower quality and lower cost electronics (RF, ADC and DSP resolution). Therefore, we believe that the sensitivity of the gateway may be significantly improved, leading to the coverage extension. Note that the advertized Texas Instruments CC2530 receiver sensitivity is approximately $-95 \mathrm{dBm}$, significantly outperforming the basic 802.15.4 requirement.

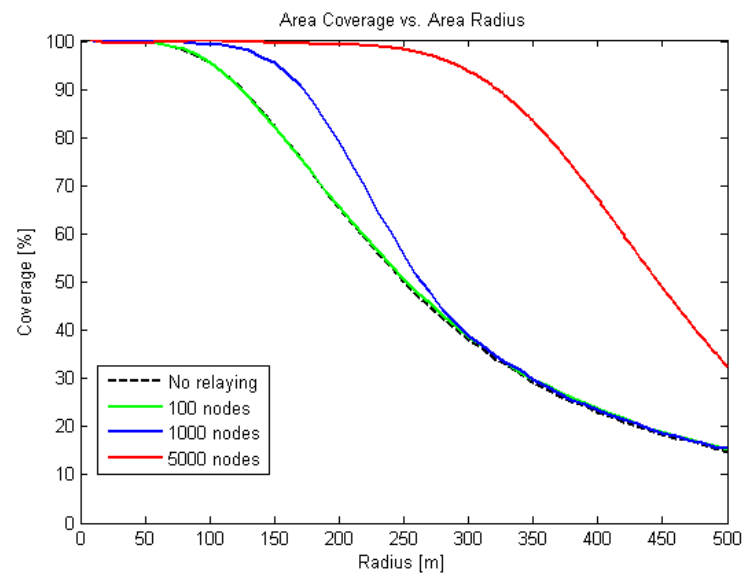

Figure 11. Multi-hop coverage as a function of area radius and number of nodes, i.e., node density.

Table II. Multi-hop coverage.

\begin{tabular}{|c|c|c|}
\hline & $90 \%$ coverage & $99 \%$ coverage \\
\hline No relaying & $125 \mathrm{~m}$ & $80 \mathrm{~m}$ \\
\hline 100 nodes & $125 \mathrm{~m}$ & $80 \mathrm{~m}$ \\
\hline 1000 nodes & $175 \mathrm{~m}$ & $125 \mathrm{~m}$ \\
\hline 5000 nodes & $325 \mathrm{~m}$ & $230 \mathrm{~m}$ \\
\hline
\end{tabular}

In addition, application of directional antennas on the gateway side could further improve the coverage. These are the topics of out ongoing and future research.

\section{CONCLUSIONS}

In this paper we have presented Road Nail, an intelligent road marking system with the wireless network of signaling devices that are mounted near the road edge. The signaling devices are autonomous nodes with capability to accumulate electrical energy into battery which is obtained from solar panels. In addition, the nodes exchange wireless messages, detect approaching vehicles and emit signalization light.

We have described our experimental testbed that consists of 20 nodes and a cellular gateway. The implementation details of the above system, including extensive measurements and performance evaluations in realistic field deployments are reported. Performance of the PIR and light sensor, power consumption of each subsystem, as well as, the solar cell power generation has been provided.

A detailed gateway-to-node coverage analysis was presented. The results indicate very limited baseline coverage with potential gains due to multi-hopping, i.e., relaying between the nodes. Furthermore, the receiver sensitivity gap points to potentially sizable gains.

We believe that application of the presented V2I system would dramatically improve the traffic road safety.

\section{REFERENCES}

[1] D. B. Rawat, D. Treeumnuk, D. C. Popescu, M. Abuelela, and S. Olariu, "Challenges and perspectives in the implementation of the NOTICE architecture for vehicular communications,” the 2nd IEEE Workshop on Mobile Vehicular Networks (MoVeNet 2008), 2008.

[2] D. Treeumnuk, D. C. Popescu, and S. Olariu, "Requirements for the physical layer of the NOTICE system for vehicular communications," IEEE Global Telecomunications, 2009.

[3] Michael Chui, Markus Löffler, and Roger Roberts, "The Internet of things," McKinsey Quarterly 2010.

[4] The World Fact Book, CIA, 2010.

[5] IEEE Std 802.15.4 - 2003, IEEE Computer Society, Oct. 2003.

[6] IST-WINNER II Deliverable 1.1 .2 v.1.2, "WINNER II Channel Models," IST-WINNER2, 2007.

[7] T. Fujii, "Path-loss prediction formula in mobile communication-an expansion of "SAKAGAMI" path-loss prediction formula," Trans. IEICE, Japan, J86-B, 10, pp. 2264 2267, 2003.

[8] "Proposed Propagation Models for Evaluation Radio Transmission Technologies in IMT Advanced," Doc. 5D/88, January 2008, Geneva, Switzerland.

[9] A. Fanimokun, and J. Frolik, "Effects of natural propagation environments on wireless sensor network coverage area," Proceedings of the 35th Southeastern Symposium on System Theory, 2003. 